\title{
Multi-Fidelity Surrogate-Based Parameter Estimation for a Sailing Yacht Hull
}

\author{
$\underline{\text { Jouke H.S. de Baar }}^{\text {a }}$, Stephen G. Roberts ${ }^{\mathrm{b}}$ \\ ${ }^{a}$ Mathematical Sciences Institute, The Australian National University, John Dedman Building 27, Union \\ Lane, Canberra ACT 2601, Australia \\ ${ }^{\mathrm{b}}$ Mathematical Sciences Institute, The Australian National University, John Dedman Building 27, Union \\ Lane, Canberra ACT 2601, Australia \\ Email: j.h.s.debaar@gmail.com
}

\begin{abstract}
Given a numerical simulation $m: \boldsymbol{\xi} \mapsto \boldsymbol{y}_{\text {num }}$, the objective of parameter estimation is to provide a joint posterior probability distribution $p\left(\boldsymbol{\xi} \mid \boldsymbol{y}_{\text {exp }}\right)$ for an uncertain input parameter vector $\boldsymbol{\xi} \in \mathbb{R}^{d}$, conditional on available experimental data $\boldsymbol{y}_{\exp } \in \mathbb{R}^{q}$. However, exploring the posterior requires a high number of numerical simulations, which can make the problem impracticable within a given computational budget.
\end{abstract}

A well-known approach to reduce the number of required simulations is to construct a surrogate, which based on a set of training simulations - can provide a inexpensive approximation of the simulation output for any parameter configuration.

To further reduce the total cost of the simulations, we can introduce low-fidelity as well as high-fidelity training simulations. In this case, a small number of expensive high-fidelity simulations is augmented with a larger number of inexpensive low-fidelity simulations. We investigate the scaling of the computational cost with the number of parameters, as well as the optimal ratio of the number of low-fidelity and high-fidelity training simulations.
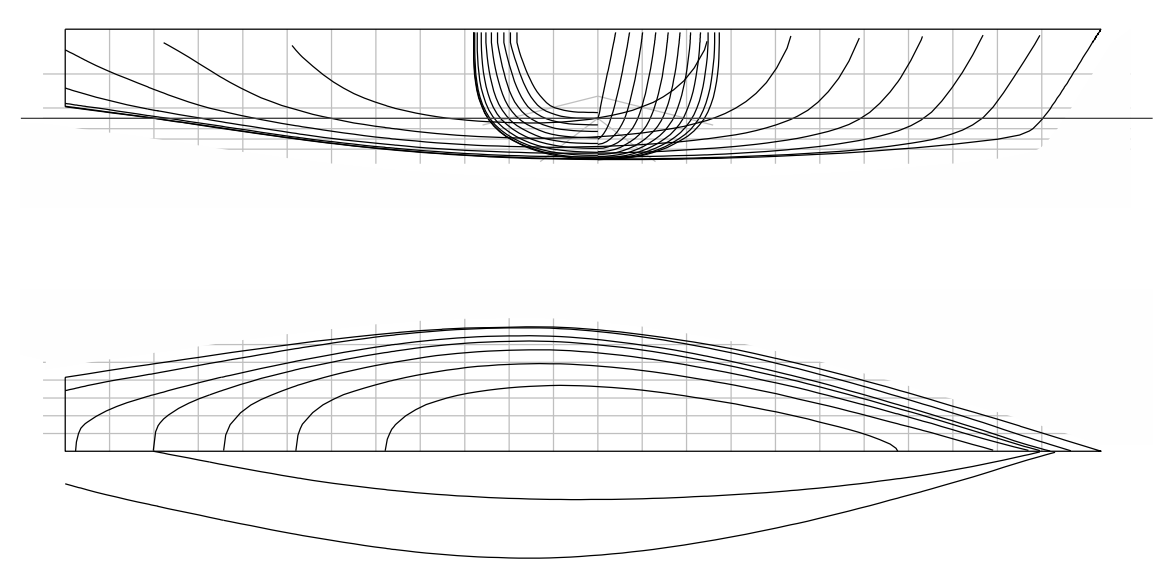

Figure 1. Lines plan of the sailing yacht hull used for multi-fidelity surrogate-based parameter estimation, showing the sections, buttocks, waterlines and diagonal cuts.

As an application we consider a towing tank experiment of the sailing yacht hull shown in Figure 1. The highfidelity and low-fidelity simulations $m_{\mathrm{HF}}$ and $m_{\mathrm{LF}}$ solve the free-surface Reynolds-averaged Navier-Stokes equations on high and low-resolution grids, respectively. Experimental data $\boldsymbol{y}_{\text {exp }}$ are available for the resistance, sinkage and pitch over a range of Froude numbers. The uncertain parameters $\boldsymbol{\xi}$ are the tank blockage, the mass and the centre of gravity. As a result we conclude that the centre of gravity is very close to the value provided by the laboratory, and that the tank blockage and mass are negatively correlated.

Keywords: Parameter estimation, inverse problem, surrogate, multi-fidelity, free-surface, Reynolds-averaged Navier-Stokes 
J.H.S. de Baar and S.G. Roberts, Multi-fidelity surrogate-based parameter estimation for a sailing yacht hull

\section{INTRODUCTION}

The objective of this investigation is to use experimental data to estimate the uncertain input parameters (tank blockage, mass and centre of gravity) of a sailing yacht hull. To reduce the number of required simulations, we first create a surrogate of the output of the computer simulation. Once the surrogate is available, we can evaluate it at relatively low cost, which enables us to estimate the parameters without further solver evaluations.

In Section 2 we discuss Kriging and multi-fidelity Kriging surrogate modelling. In Section 3 we investigate the performance of multi-fidelity Kriging for an analytical test-function. In Section 4, we apply multi-fidelity Kriging to estimate the parameters of the sailing yacht hull.

\section{Method}

In Section 2.1 we discuss parameter estimation using Bayes' Rule, where our approach is to explore the likelihood at lower cost by approximating the simulation output with a surrogate. In Sections 2.2 and 2.3 we discuss Kriging and multi-fidelity Kriging, which we will use as the surrogate.

\subsection{Surrogate-based parameter estimation}

Given experimental data $\boldsymbol{y}_{\exp } \in \mathbb{R}^{q}$ and uncertain parameter $\boldsymbol{\xi} \in \mathbb{R}^{d}$ with prior probability density $p(\boldsymbol{\xi})$, we use Bayes’ Rule (Bayes, 1763; Laplace, 1774):

$$
p\left(\boldsymbol{\xi} \mid \boldsymbol{y}_{\exp }\right) \propto p\left(\boldsymbol{y}_{\exp } \mid \boldsymbol{\xi}\right) p(\boldsymbol{\xi}),
$$

to obtain the posterior distribution of the uncertain parameters, conditional on the experimental data. We assume an unbiased, uncorrelated normal likelihood, given by:

$$
\boldsymbol{y}_{\exp } \mid \boldsymbol{\xi} \sim \mathcal{N}(m(\boldsymbol{\xi}), \Sigma)
$$

The likelihood models the experimental process: given a choice of the uncertain parameters $\boldsymbol{\xi}$, the simulation $m: \mathbb{R}^{d} \rightarrow \mathbb{R}^{q} ; \boldsymbol{\xi} \mapsto \boldsymbol{y}_{\text {num }}$ acts as the expected value of the experimental data $\boldsymbol{y}_{\text {exp }}$, while $\Sigma$ is the $q \times q$ experimental error covariance matrix.

Solving the inverse problem by exploring (1) is expensive, as it requires multiple evaluations of the solver $m$ in (2), at high computational cost of up to hours or days for an individual solve, to the extent of making the solution of the problem impracticable. Our approach is to create a surrogate $\tilde{m}$ of $m$, which, based on a set of training simulations, provides a statistical estimate of the output of the numerical solver (Sacks et al., 1989). In the next subsections, we discuss the construction of an accurate surrogate.

\subsection{Kriging}

To construct a surrogate $\tilde{m}$ we introduce a process $\boldsymbol{X} \in \mathbb{R}^{n}$, which represents the ideal simulation output. We model the covariance of $\boldsymbol{X}$ by the normally distributed prior:

$$
\boldsymbol{X} \sim \mathcal{N}(\boldsymbol{\mu}, P)
$$

with mean $\boldsymbol{\mu} \in \mathbb{R}^{n}$ and $n \times n$ covariance matrix $P$, which is a function of $\xi$. We run $N$ numerical experiments to obtain training data $\boldsymbol{y}_{\text {train }} \in \mathbb{R}^{r}$, where $r=N q$, and we now note that $n=q+r$. We assume the unbiased and uncorrelated likelihood:

$$
\boldsymbol{y}_{\text {train }} \mid \boldsymbol{X} \sim \mathcal{N}(H \boldsymbol{X}, R),
$$

with $r \times n$ observation matrix $H$, which selects the training data, and $r \times r$ observation error covariance matrix $R$. Using Bayes' Rule we define the surrogate as the expectation of the process $\boldsymbol{X}$ (Matheron, 1963; Gandin, 1965; Cressie, 1993; Wikle and Berliner, 2007):

$$
\begin{aligned}
\tilde{m}(\boldsymbol{\xi}) & =\mathrm{E}\left(Q \boldsymbol{X} \mid \boldsymbol{y}_{\text {train }}\right) \\
& =Q \boldsymbol{\mu}+Q K_{\boldsymbol{\xi}}\left(\boldsymbol{y}_{\text {train }}-H \boldsymbol{\mu}\right),
\end{aligned}
$$

where we have the $n \times r$ Kalman gain $K_{\xi}=P H^{T}\left(R+H P H^{T}\right)^{-1}$ and the $q \times n$ prediction matrix $Q$, which selects the experimental data. We note that the covariance matrix $P$ depends on the input parameters $\boldsymbol{\xi}$, as we generate $P$ from a Gaussian covariance function, where we find the correlation lengths from a maximum likelihood estimate (Mardia and Marshall, 1984). The conditional covariance $\operatorname{cov}\left(Q \boldsymbol{X} \mid \boldsymbol{y}_{\text {train }}\right)$ is not used in this paper but can be found in (Wikle and Berliner, 2007). 
J.H.S. de Baar and S.G. Roberts, Multi-fidelity surrogate-based parameter estimation for a sailing yacht hull

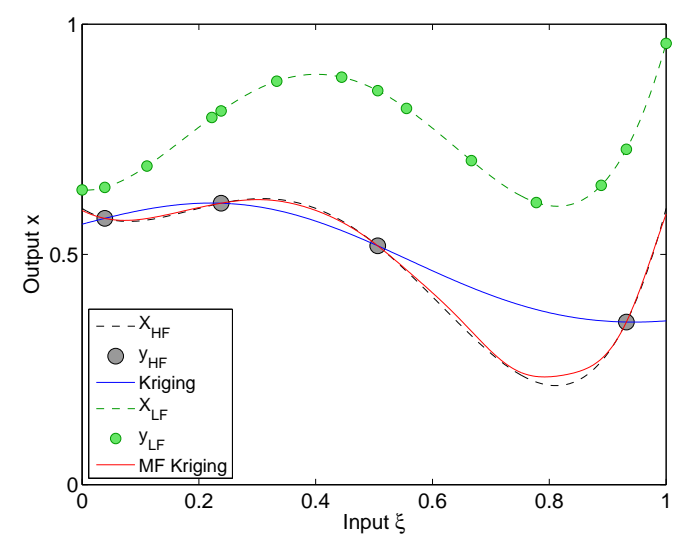

Figure 2. A low number of high-fidelity simulations can lead to an inaccurate surrogate. Adding lowfidelity simulations improves the quality of the surrogate.

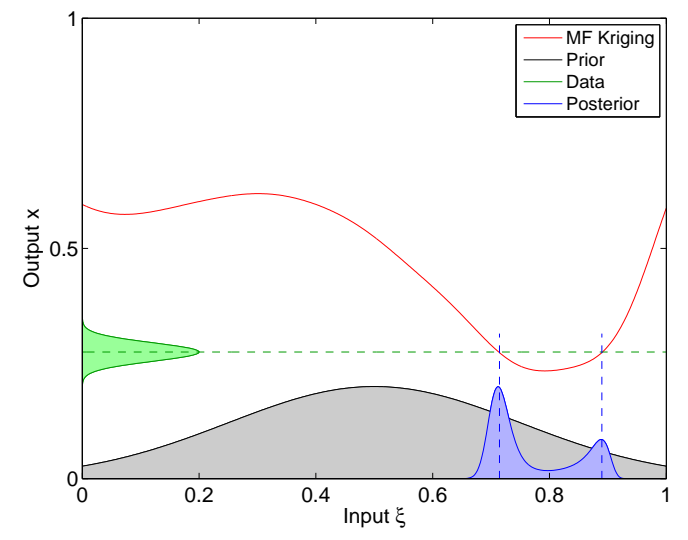

Figure 3. Surrogate-based Bayesian parameter estimation, using the prior $p(\xi)$ and the likelihood $p(d \mid \xi)$ to obtain the posterior $p(\xi \mid d)$. Note that the pdfs have been scaled.

\subsection{Multi-fidelity Kriging}

The objective of multi-fidelity Kriging is to augment a small number of high-fidelity observations $\boldsymbol{y}_{\mathrm{HF}}$ with a large number of low-fidelity observations $\boldsymbol{y}_{\mathrm{LF}}$. The assumption is that:

$$
\boldsymbol{X}_{\mathrm{HF}}=\boldsymbol{X}_{\mathrm{LF}}+\boldsymbol{X}_{\mathrm{D}}
$$

with high-fidelity process $\boldsymbol{X}_{\mathrm{HF}}$, low-fidelity process $\boldsymbol{X}_{\mathrm{LF}}$ and difference process $\boldsymbol{X}_{\mathrm{D}}$. This approach is useful if $\boldsymbol{X}_{\mathrm{D}}$ is much smoother than $\boldsymbol{X}_{\mathrm{HF}}$. The multi-fidelity Kriging predictor is given by (Kennedy and O'Hagan, 2000; Forrester et al., 2007; de Baar et al., 2015):

$$
\tilde{m}_{\mathrm{MF}}(\boldsymbol{\xi})=\boldsymbol{\mu}_{\mathrm{HF}}+K_{\mathrm{MF}}\left(\boldsymbol{y}_{\mathrm{MF}}-\boldsymbol{\mu}_{\mathrm{MF}}\right),
$$

where $\boldsymbol{y}_{\mathrm{MF}}=\left(\boldsymbol{y}_{\mathrm{LF}}, \boldsymbol{y}_{\mathrm{HF}}\right)^{T}$ and the gain:

$$
K_{\mathrm{MF}}=H_{\text {select }} P_{\mathrm{MF}} H_{\mathrm{MF}}^{T}\left(R+H_{\mathrm{MF}} P_{\mathrm{MF}} H_{\mathrm{MF}}^{T}\right)^{-1},
$$

with:

$$
H_{\mathrm{mf}} P_{\mathrm{mf}} H_{\mathrm{mf}}^{T}=\left(\begin{array}{cc}
H_{\mathrm{LF}} P_{\mathrm{LF}} H_{\mathrm{LF}}^{T} & H_{\mathrm{LF}} P_{\mathrm{LF}} H_{\mathrm{HF}}^{T} \\
H_{\mathrm{HF}} P_{\mathrm{LF}} H_{\mathrm{LF}}^{T} & H_{\mathrm{HF}} P_{\mathrm{LF}} H_{\mathrm{HF}}^{T}+H_{\mathrm{HF}} P_{\mathrm{D}} H_{\mathrm{HF}}^{T}
\end{array}\right),
$$

and:

$$
H_{\text {select }} P_{\mathrm{MF}} H_{\mathrm{MF}}^{T}=\left(H_{x} P_{\mathrm{LF}} H_{\mathrm{HF}}^{T} \quad P_{\mathrm{LF}} H_{\mathrm{HF}}^{T}+P_{\mathrm{HF}} H_{\mathrm{HF}}^{T}\right) .
$$

Here we have deviated from (Kennedy and O'Hagan, 2000; Forrester et al., 2007) by assuming that $\rho=1$.

Figure 2 illustrates the effect of including the low-fidelity simulations for a simple test-function. Clearly, including the low-fidelity data improves the accuracy of the Kriging prediction. Using the same multi-fidelity surrogate, Figure 3 illustrates surrogate-based parameter estimation. Note that the posterior can be multimodal. This corresponds to the observation that the deterministic approach does not have a unique solution.

\section{RESULTS FOR A TEST-FUNCTION}

As seen from the approximation $m \approx \tilde{m}$ in the likelihood (2), surrogate-based parameter estimation requires an accurate surrogate, which can be constructed at low computational cost. A key performance indicator is the number of solves that are required to achieve a certain target accuracy $\delta$, such that the surrogate prediction error $\sqrt{\left.\left\{\tilde{m}_{\mathrm{MF}}(\boldsymbol{\xi})-\boldsymbol{X}\right\}^{2}\right]}<\delta$. This indicator is known as the number of 'solves to target'.

Using a test-function, we investigate the effect on the number of solves to target for two important variables:

(1) the optimimal ratio between the number of low-fidelity and high-fidelity and (2) the computational speedup 
J.H.S. de Baar and S.G. Roberts, Multi-fidelity surrogate-based parameter estimation for a sailing yacht hull

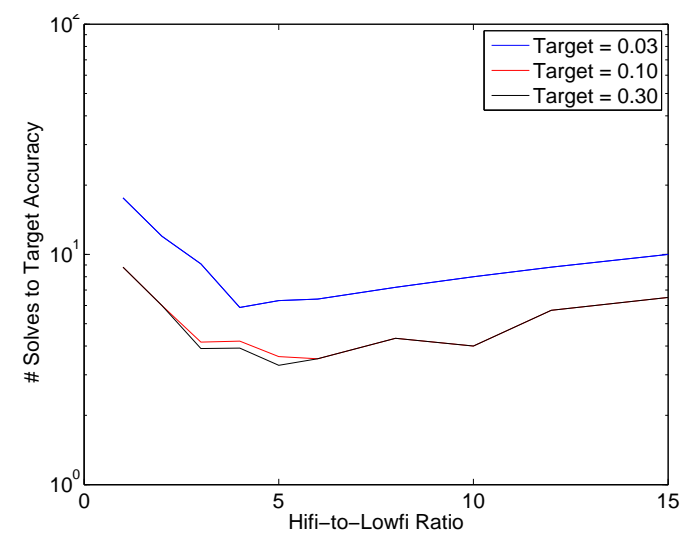

Figure 4. The optimal lowfi-to-hifi ratio depends on the target accuracy (2-dimensional case).

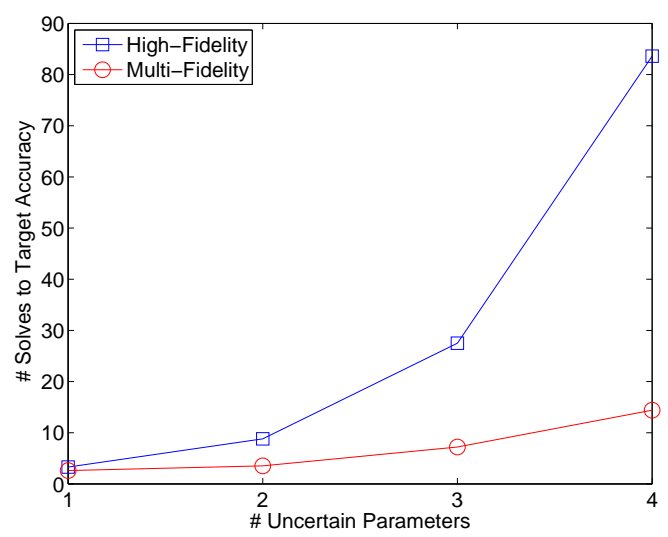

Figure 6. The number of high-fidelity solves required to produce a surrogate of a given target accuracy increases with the number of parameters. Including low-fidelity solves reduces this effect.

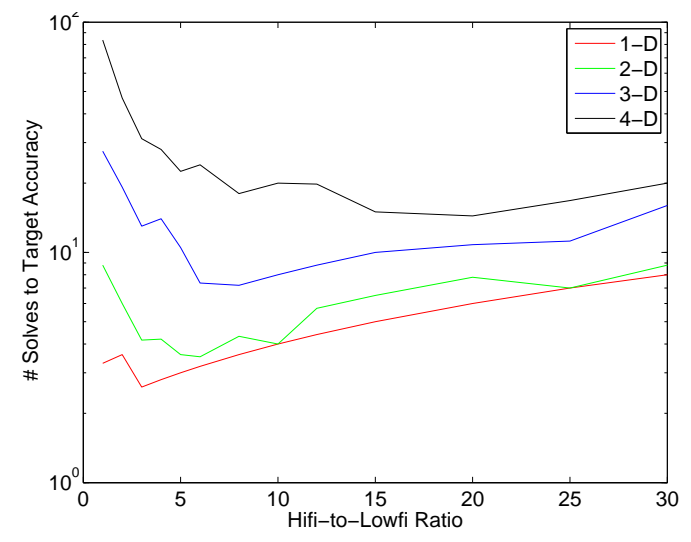

Figure 5. The optimal lowfi-to-hifi ratio depends on the number of parameters.

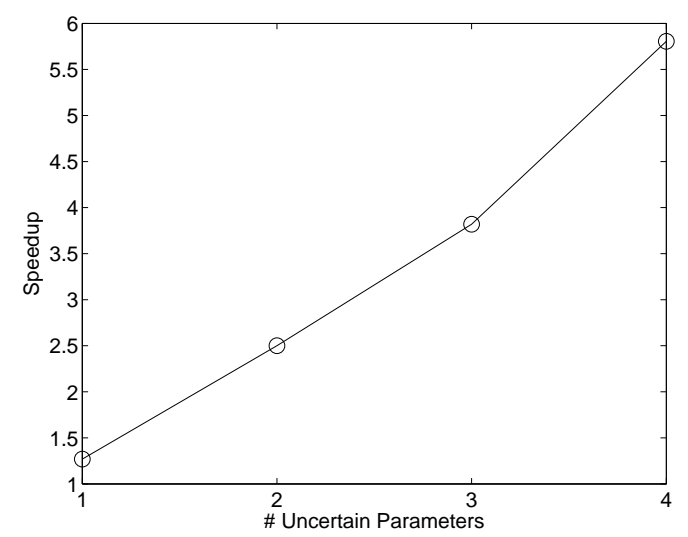

Figure 7. The speedup, which is defined as the ratio of the cost of the high-fidelity and multi-fidelity surrogates, increases with the number of parameters.

we can expect for an increasing number of input parameters. As the response of many engineering simulations is smooth, we choose a smooth test-function:

$$
\begin{aligned}
m_{\mathrm{HF}}(\boldsymbol{\xi}) & =\sum_{k=1}^{d} \cos \left(\frac{4}{5} \pi \xi_{k}\right) \\
m_{\mathrm{LF}}(\boldsymbol{\xi}) & =\sum_{k=1}^{d} \cos \left(\frac{4}{5} \pi \xi_{k}\right)+\sum_{k=1}^{d} \sin \left(\frac{1}{5} \pi \xi_{k}\right)
\end{aligned}
$$

for the high-fidelity and low-fidelity process, respectively, for a $d$-dimensional parameter space. We further assume that the cost of a high-fidelity simulation is 10 times that of a low-fidelity simulation. All results are averaged over 5 different space-filling sampling plans (Forrester et al., 2008).

\subsection{Lowfi-to-hifi ratio}

The first important question is: what is the optimal ratio of the number of low-fidelity to high-fidelity simulations? This ratio is likely to be influenced by the required target accuracy of the surrogate, as well as by the number of dimensions.

Figure 4 shows the effect of the lowfi-tho-hifi ratio on the total cost (measured in high-fidelity simulations) to achieve various relative target accuracies in the two-dimensional case. In general, it is more expensive to reach a higher accuracy, however, the optimal lowfi-to-hifi ratio appears to be at a constant value. 
J.H.S. de Baar and S.G. Roberts, Multi-fidelity surrogate-based parameter estimation for a sailing yacht hull
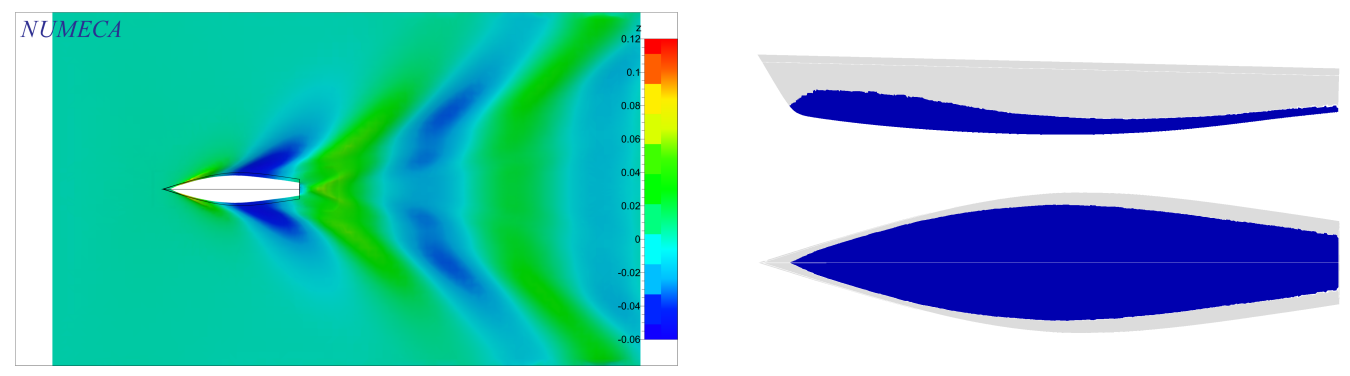

Figure 8. The free-surface elevation (left-hand side) and wetted surface (right-hand side) for a Froude number of $\mathrm{Fn}=0.49$, the highest velocity included in the calibration process.

Table 1. Input parameters $\xi$.

\begin{tabular}{lcccc}
\hline Parameter & Symbol & Mean & Std. & Unit \\
\hline Blockage & $c$ & 1.004 & 0.002 & - \\
Mass & $M$ & 36.95 & 0.07 & $\mathrm{~kg}$ \\
Centre of gravity & $x_{C o G}$ & -0.96 & 0.05 & $\mathrm{~m}$ \\
\hline
\end{tabular}

Table 2. Output uncertainties in data $y$.

\begin{tabular}{lclr}
\hline Parameter & Symbol & Std. & Unit \\
\hline Resistance & $R$ & 0.2 & $\mathrm{~N}$ \\
Sinkage & $\Delta z$ & 0.13 & $\mathrm{~mm}$ \\
Pitch & $\alpha$ & 0.05 & $o$ \\
\hline
\end{tabular}

Figure 5 shows the effect of the lowfi-tho-hifi ratio on the total cost to achieve a relative target accuracy of 0.1 , but now for an increasing number of uncertain parameters. The optimal lowfi-to-hifi ratio appears to be increasing linearly with the number of dimensions.

\subsection{Computational speedup}

Figure 6 shows how the cost of constructing an accurate surrogate, with a relative target accuracy of 0.1 , increases with the number of parameters. If we use only high-fidelity simulations, indicated by the blue line, the cost increases dramatically, an effect known as the 'curse of dimensionality'. When we use both lowfidelity and high-fidelity simulations, the overall cost, indicated by the red line, is reduced significantly.

Figure 7 shows the computational speedup, defined as the ratio of the computational cost of constructing either the high-fidelity or the multi-fidelity surrogate. The grey area indicates the standard deviation as a result averaging results over different sampling plans. The speedup appears to increase almost linearly with the number of parameters, indicating that the multi-fidelity approach becomes increasingly efficient for more higher-dimensional inverse problems.

\section{RESULTS FOR A SAILING YACHT HULL}

The Delft Systematic Yacht Hull Series (DSYHS) is a range of 70 systematically varied yacht hulls, based on a number of parent models (Kerwin, 1975; Gerritsma et al., 1981; Keuning and Katgert, 2008). We consider DSYHS hull number 25, the lines plan of this hull is shown in Figure 1 and the waterline length is $2 \mathrm{~m}$. This hull has been tested in a towing tank with a length of $142 \mathrm{~m}$, a width of $4.22 \mathrm{~m}$ and a water depth of $2.50 \mathrm{~m}$. The hull has been tested up to a Froude number of $\mathrm{Fn}=0.75$, however, we currently restrict ourselves to speeds of $\mathrm{Fn} \leqslant 0.5$.

For the numerical simulations $m$ we use FINE/Marine (Numeca, Brussels, Belgium), a free-surface ReynoldsAveraged Navier-Stokes (RANS) solver with a finite volume spatial discretisation (Duvigneau et al., 2003; Visonneau et al., 2006). We use a Menter-SST k- $\omega$ turbulence model (Menter, 1994). For the high-fidelity and low-fidelity simulations, we use grids of 380,000 and 42,000 cells respectively. Because a change in blockage is equivalent to a change in velocity, we introduce the velocity as a dummy parameter. The solution for $\mathrm{Fn}=0.49$ is illustrated in Figure 8, showing the typical high bow wave as well as a wave length which is close to the hull length.

Based on expert opinion, we consider three uncertain input parameters $\xi$ : the tank blockage $c$, the hull mass $M$ and the lengthwise position of the centre of gravity $x_{\mathrm{CoG}}$. The estimated mean and standard deviations are given in Table 1. Estimating the posterior of these input parameters is the main objective of this paper. The experimental output uncertainties in the data $y$ are estimated in Table 2. Most of the estimated input and output uncertainties are based on ITTC (2002) and ITTC (2011).

To construct the surrogate, we use a use a space-filling design (Forrester et al., 2008) to create a set of training data of 24 high-fidelity and 120 low-fidelity simulations. Without adding new simulations, we then exploit the 

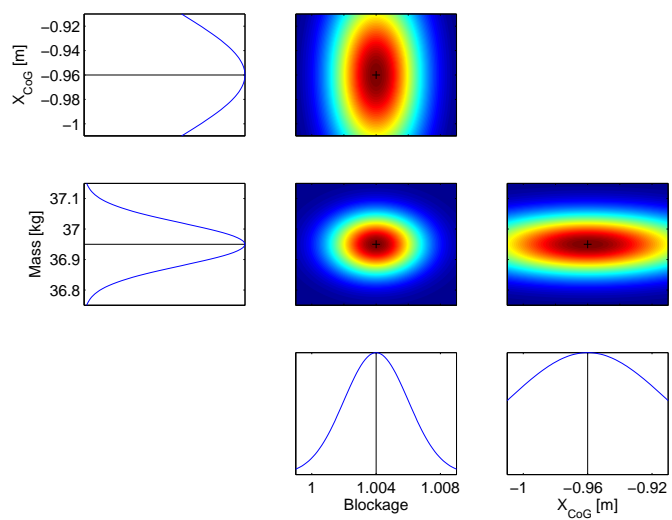

Figure 9. The prior marginal distributions of the uncertain parameters.
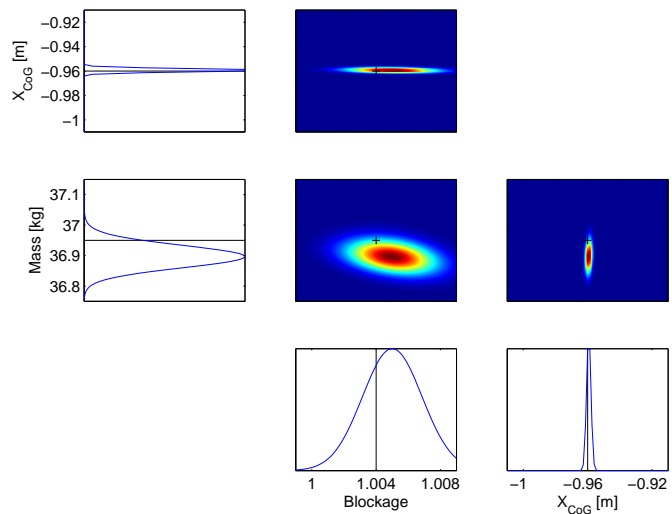

Figure 10. The posterior marginal distributions of the uncertain parameters.

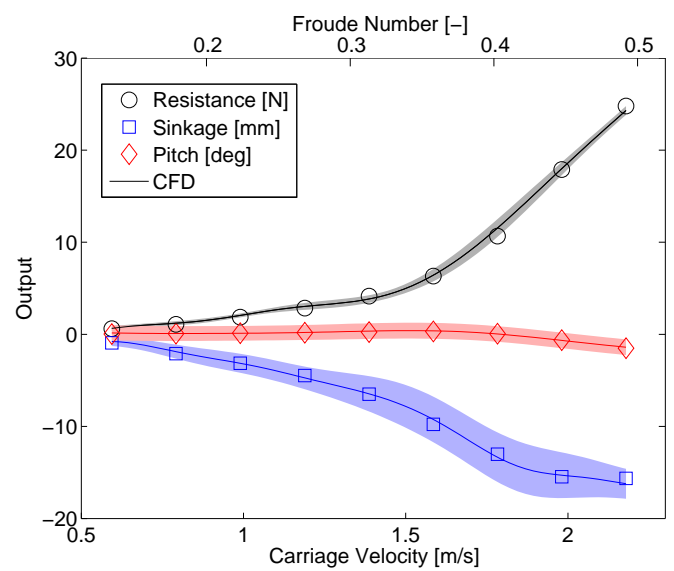

Figure 11. Experimental data and simulation results for the prior parameter distribution.

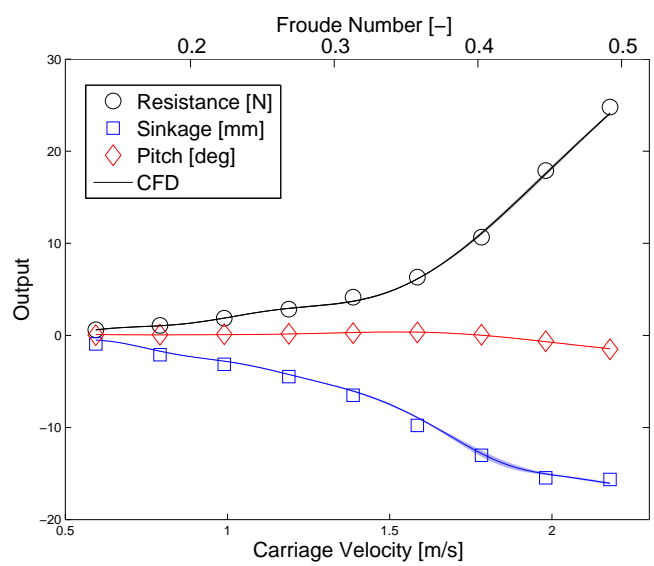

Figure 12. Experimental data and simulation results for the posterior parameter distribution.

The prior distribution of the uncertain parameters is illustrated in Figure 9. As a result of the uncertainties in the input parameters, we see large uncertainties in the corresponding simulation results in Figure 10, where the lines indicate the expected value and the shaded areas indicate the standard deviation in the simulation results as an effect of the uncertain inputs. Given the experimental data, we find the posterior parameter distribution in Figure 11. Interestingly, we see a negative correlation between the mass and the blockage, which might be explained by the fact that both quantities have the effect of increasing the resistance. The corresponding simulation results are shown in Figure 12, now with a much smaller standard deviation.

\section{CONCLUSIONS}

We have investigated the performance of multi-fidelity Kriging for a test-function. Results indicate that the lowfi-to-hifi ratio scales with the number of uncertain parameters, and that the method shows a linear speedup when the number of parameters increases. However, further investigations are necessary.

We have succesfully applied multi-fidelity Kriging to estimate three uncertain parameters — tank blockage, hull mass and centre of gravity — for a sailing yacht hull. The estimated values are close to the values given by the laboratory. 
J.H.S. de Baar and S.G. Roberts, Multi-fidelity surrogate-based parameter estimation for a sailing yacht hull

\section{ACKNOWLEDGements}

This paper contains joint work with Benoit Mallol (Numeca, Brussels, Belgium) and Richard Dwight (Delft University of Technology, Delft, The Netherlands).

\section{REFERENCES}

Bayes, T. (1763). An essay towards solving a problem in the doctrine of chances. Philosophical Transactions of the Royal Society of London (53), 370-418.

Cressie, N. (1993). Statistics for spatial data. Wiley.

de Baar, J., S. Roberts, R. Dwight, and B. Mallol (2015). Uncertainty quantification for a sailing yacht hull, using multi-fidelity Kriging. submitted.

Duvigneau, R., M. Visonneau, and G. Deng (2003). On the role played by turbulence closures in hull shape optimization at model and full scale. Journal of Marine Science and Technology (8), 11-25.

Forrester, A., A. Sobester, and J. Keane (2007). Multi-fidelity optimization via surrogate modelling. Proceedings of the Royal Society A 463, 3251-3269.

Forrester, A. I., A. Sobester, and A. J. Keane (2008). Engineering design via surrogate modelling, a practical guide. Wiley.

Gandin, L. (1965). Objective analysis of meteorological fields: Gidrometeorologicheskoe Izdatel'stvo (GIMIZ), Leningrad. Translated by Israel Program for Scientific Translations, Jerusalem.

Gerritsma, J., R. Onnink, and A. Versluis (1981). Geometry, resistance and stability of the Delft systematic yacht hull series. 7-th HISWA Symposium, 1981, Amsterdam.

ITTC (2002). Recommended procedures: Resistance uncertainty analysis, example for resistance test. International Towing Tank Committee Report 7.5-02-02-02.

ITTC (2011). Recommended procedures and guidelines: Resistance test. International Towing Tank Committee Report 7.5-02-02-01.

Kennedy, M. C. and A. O'Hagan (2000). Predicting the output from a complex computer code when fast approximations are available. Biometrika $87,1-13$.

Kerwin, J. (1975). A velocity prediction program for ocean racing yachts. Report 78-11, Department of Ocean Engineering, Massachusetts Institute of Technology.

Keuning, J. and M. Katgert (2008). A bare hull resistance prediction method derived from the results of the Delft systematic yacht hull series extended to higher speeds. International Conference on Innovation in High Performance Sailing Yachts, Lorient, France.

Laplace, P.-S. (1774). Mémoire sur la probabilité des causes par les événements. Mémoires de Mathématique et de Physique Présentés a l'Académie Royale des Sciences (6), 621-656.

Mardia, K. V. and R. J. Marshall (1984). Maximum likelihood estimation of models for residual covariance in spatial regression. Biometrika 71(1), 135-146.

Matheron, G. (1963). Principles of Geostatistics. Economic Geology 58, 1246-1266.

Menter, F. (1994). Two-equation eddy-viscosity turbulence models for engineering applications. AIAA Journal 32(8), 1598-1605.

Sacks, J., W. J. Welch, T. J. Mitchell, and H. P. Wynn (1989). Design and analysis of computer experiments. Statistical Science 4(4), 409-423.

Visonneau, M., P. Queutey, and G. Deng (2006). Model and full-scale free-surface viscous flows around fully-appended ships. European Conference on Computational Fluid Dynamics, ECCOMAS CFD.

Wikle, C. K. and L. M. Berliner (2007). A Bayesian tutorial for data assimilation. Physica D: Nonlinear Phenomena 230(1-2), 1 - 16. 